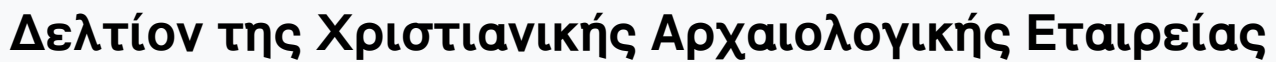

Tó 30 (2009)

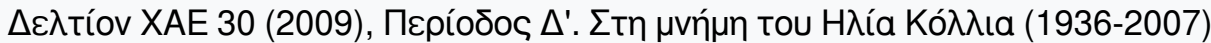

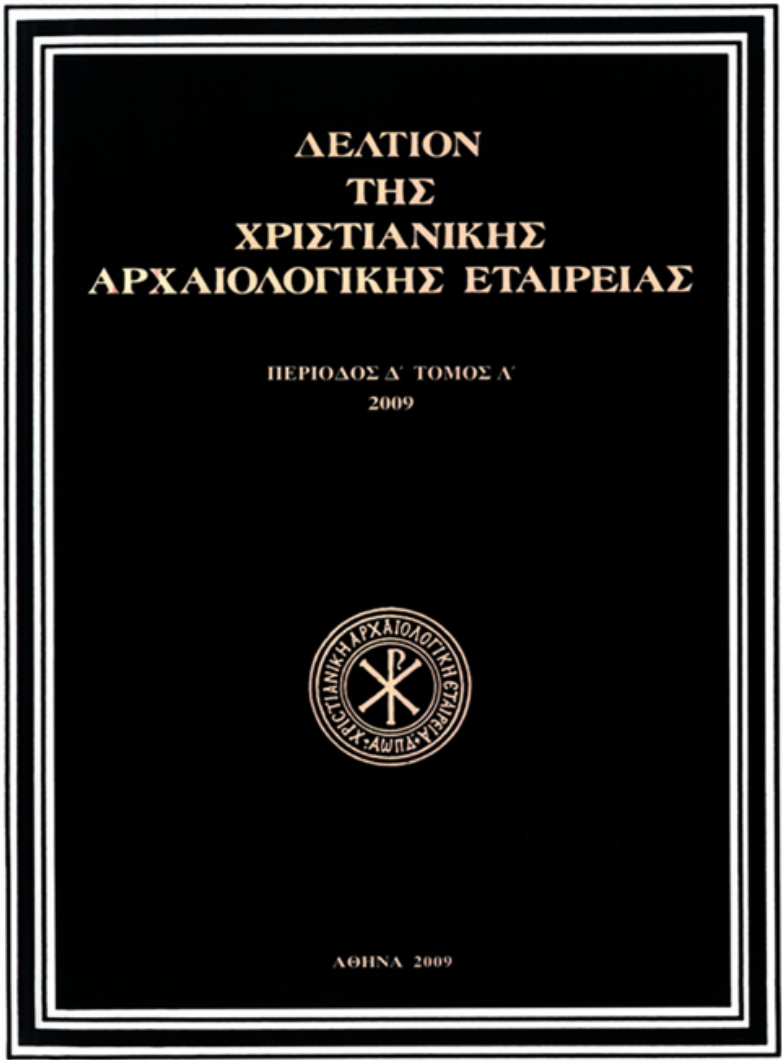

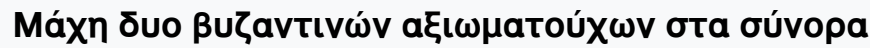

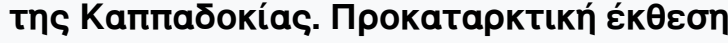

Nicole THIERRY

doi: $\underline{10.12681 / \text { dchae. } 647}$

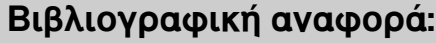

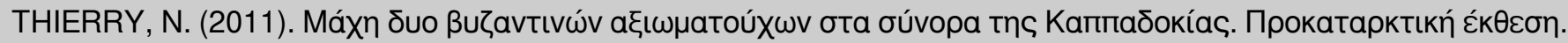

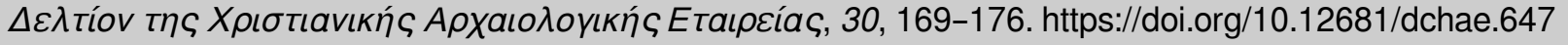




\section{$\triangle \mathrm{E} \Lambda \mathrm{TION}$ TH $\Sigma$ XPI $\Sigma$ TIANIKH $\Sigma$ APXAIO $\Lambda$ OГIKH $\Sigma$ ETAIPEIA $\Sigma$}

Portraits funéraires inédits de deux officiers byzantins morts au combat sur les frontières de la Cappadoce. Étude préliminaire

Nicole THIERRY

Пe@íodos $\Delta^{\prime}$, Tómos $\Lambda^{\prime}(2009) \bullet \Sigma \varepsilon \lambda$. 169-176 A@HNA 2009 


\author{
Nicole Thierry
}

\title{
PORTRAITS FUNÉRAIRES INÉDITS DE DEUX OFFICIERS BYZANTINS MORTS AU COMBAT SUR LES FRONTIÈRES DE LA CAPPADOCE ÉTUDE PRÉLIMINAIRE
}

$\mathrm{C}$ ette image se trouve au village de Gelvere (Güzelyurt), la Karbala de Grégoire de Nazianze, dans une chapelle funéraire rupestre effondrée ${ }^{1}$.

Elle représente une scène de combat entre le démon et deux cavaliers, un skribone Léon et un tourmarque Michel. Elle est peinte au-dessus d'un grand tombeau encore plein de terre (Fig. 3). Il s'agit d'un hapax, bien qu'on reconnaisse la composition populaire des saints affrontés, Georges et Théodore, perçant le serpent de leur lance, et, particulièrement, celle d'Yılanlı kilise du vallon voisin d'Ihlara où les deux têtes sont frappées. Une croix est plantée dans le Diable qui reconnaît sa défaite: «Croix, qui t'a fait briller ? Le Christ qui est fiché en moi » (Fig. 8) ${ }^{2}$.

Notre découverte fut fortuite, au retour d'une visite des montagnes du Melendiz qui, au sud, faisaient partie de la seconde frontière byzantine après celle du Taurus, du temps des guerres arabo-byzantines (Fig. 1). Les villageois, qui nous ont guidés pour retrouver les ruines de Drizion, résidence où Nicéphore Phocas avait installé la famille impériale durant ses campagnes syriennes de 964 et $965^{3}$, nous ont

\footnotetext{
${ }^{1}$ Église du quartier dit Așağı mahallı, découverte en septembre 2006 par Pierre Lucas ; située sur les terres patrimoniales de Grégoire, où il se retira après avoir quitté son siège de Constantinople. Ses reliques, conservées dans la grande église du village, ont été transférées en Grèce après 1924, et depuis peu, dans la nouvelle église de Nea Karvali. Pour Karbala, cf. F. Hild, M. Restle, Kappadokien, TIB 2, Vienne 1981, 203204 (cité TIB 2).

${ }^{2}$ N. Thierry, « Aux limites du sacré et du magique. Un programme d'entrée d'une église en Cappadoce », Res Orientales XII (1999), 33-247. Ihlara est à $6 \mathrm{~km}$ au sud-ouest ( $c f$. Fig. 1).

${ }^{3}$ F. Hild, «Il sistema viario della Cappadocia », in Le aree omogene della Civiltà Rupestre : la Cappadocia, Galatino 1981, 115-123. TIB 2, 172173. Nous avions supposé leur disparition, tant les paysans semblaient les ignorer. N. Thierry, La Cappadoce de l'Antiquité au Moyen Âge, Turnhout 2002, 15, n. 18 (cité La Cappadoce). Depuis Friedrich Hild
}

signalé en montagne, au-dessus de Yeşilyurt, un château différent de la fortification voisine du village de Çömlekçi connue depuis peu sous le nom de Koron ${ }^{4}$.

Il se trouve plus à l'est, sur le Kale Tepesi de la carte au $1 / 200.000$ e de 1951 , à près de $2200 \mathrm{~m}$ d'altitude. Des ruines sur une plate-forme, de longs fragments de murailles au pied de la paroi, et tout un système de tunnels et d'escaliers taillés dans le roc, permettent d'y reconnaître plutôt Koron, la célèbre place forte, lieu de séjour du stratège byzantin jusqu'à la fin du IXe siècle, et plusieurs fois prise et reprise par les Arabes jusqu'en $897^{5}$. Le fort proche de Çömlekçi est nommé Korunun başı (la tête de Koron) ; il pourrait s'agir d'un poste-sentinelle au-dessus de la plaine de Drizion, en avant-garde de la citadelle, bien en retrait en haute montagne. Je reviendrai ailleurs sur ce district de Qurra (Tabari), que les Arabes investissaient lorsqu'ils déferlaient par les Portes de Cilicie.

Du VIIIe au Xe siècle, des combats s'y livrèrent, connus par les chroniqueurs arabes et les ouvrages militaires des Takti$k a$ de Léon VI et Nicéphore Phocas ${ }^{6}$. Le pays fut divisé en

m'a amicalement envoyé des précisions topographiques dont je lui suis reconnaissante.

${ }^{4}$ Hild, op.cit. Thierry, La Cappadoce, 15, carte 8, pl. 9.

5 TIB 2, bibliographie p. 216-217, avec étude critique d'après diverses cartes. Le site correspond à peu près à celui nommé Küre sur la carte de Kiepert (1854), suivi par O. B. R. Honigmann, Die Ostgrenze des byzantinischen Reiches von 363 bis 1071, Bruxelles 1935, carte II ; et par nous, N. et M. Thierry, Nouvelles églises rupestres de Cappadoce, Paris 1963, 10-15, carte (cité Nouvelles églises). Ses constantes GPS permettent de le retrouver sur les cartes de Google Earth.

${ }^{6}$ A. A. Vasiliev, Byzance et les Arabes, I et II (dans TIB 2, 37-38). G. Dagron et $\mathrm{H}$. Mihaescu, Le traité sur la guérilla (De velitatione) de l'empereur Nicéphore Phocas (963-969), texte établi, traduit et commenté. Appendice de J.-C. Cheynet, Les Phocas, Paris 1986. Sur l'organisation permanente des postes de guet, ch. I du texte, p. 36-38; commentaires, 245-246. 


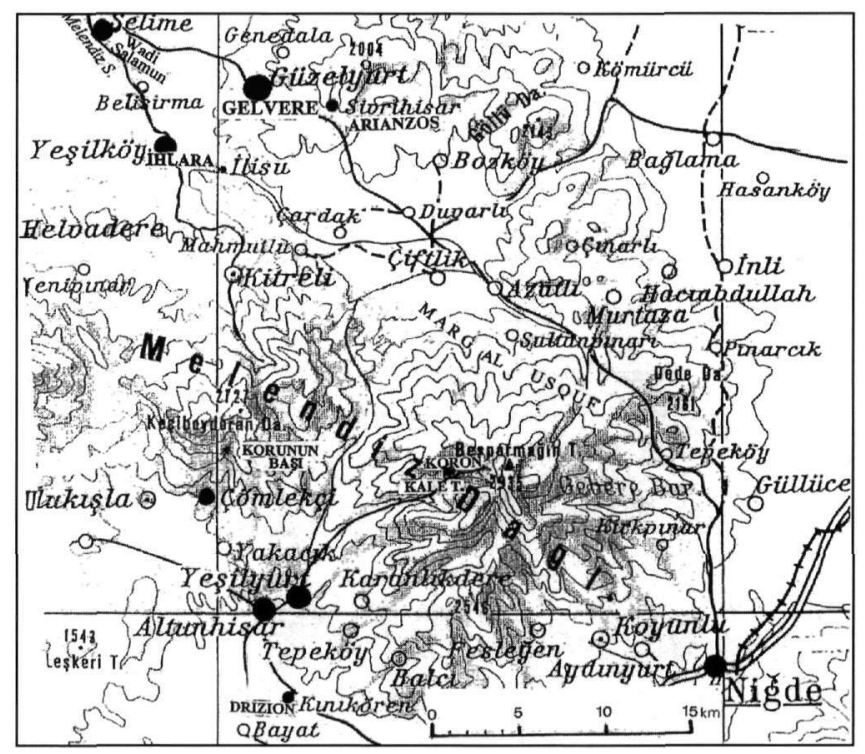

Fig. 1. Carte des Melendiz dağları à Gelvere (N. Thierry, à partir de H. Reschenhofer, TIB 2).

petits thèmes, subdivisés en tourmes, sous la responsabilité de stratèges et tourmarques, bien intégrés à la population locale à laquelle s'étaient progressivement mêlés des Arméniens et des émigrés très imprégnés de culture arabe ${ }^{7}$.

Ces rappels de géographie historique sont nécessaires pour comprendre la peinture de Gelvere, à moins de $30 \mathrm{~km}$ au nord de Koron et des zones de combat.

Elle est le seul témoin iconographique de l'hommage rendu aux héros byzantins.

\section{Description générale}

La chapelle est sous le sol devant un habitat rupestre. On y pénètre par un trou donnant sur l'entrée de l'abside. Celle-ci

\footnotetext{
${ }^{7}$ La coexistence des peuples de l'Anatolie orientale chrétienne et du monde islamique, et les modes de vie comparables des deux aristocraties affrontées, se retrouvent dans la première partie de l'épopée byzantine de Digénis Acritas, fils chrétien d'un émir arabe qui enleva la fille d'un riche stratège de Cappadoce, avec l'entente des deux familles : Corinne Jouanno, Digénis Acritas, le héros des frontières, Turnhout 1998 (33-69, à propos des nouveaux thème et du développement de régionalismes). N. Oikonomidis, «L'Épopée de Digénis et la frontière orientale de Byzance au Xe et XIe siècles », TM 7 (1979), 375-397.

${ }^{8}$ Cf. celle de saint David, J.-M. Thierry de Crussol, Monuments arméniens de Haute-Arménie, CNRS Paris 2005, 54, fig. 13.
}

et la partie nord du monument sont écrasées (Fig. 7). À gauche subsistent deux arcades séparées par un pilier. Seule la première est peinte.

Lors de notre visite de 2006, le sol de la nef était intact. Depuis, des villageois ont dégagé un autre tombeau au pied du premier, et ouvert une fosse profonde. Au pied du tombeau inférieur, vers l'ouest, on voit une niche cubique taillée dans le pilier (Fig. 7). Il est possible qu'il s'agisse d'une " confessio » 8 . En 2008, j'appris du Kaymakan ${ }^{9}$ de la ville d'Ortaköy (l'antique Antigus) que des fouilleurs clandestins avaient été arrêtés. L'architecture de cette église rupestre fut soignée ; une corniche court sur les piliers, à l'appui des arcades et sur la paroi du fond (Fig. 3). Un enduit de mortier blanc très dur a revêtu les parois restées en partie sans peinture. Au fond de la nef, deux panneaux hagiographiques encadraient l'arc de la niche centrale. Il n'en reste que le buste abîmé d'un saint. L'arcade sud-est abrite le grand tombeau-sarcophage surmonté du panneau figuratif sous plafond (Fig. 7). Le couvercle brisé a été jeté sur les pierres éboulées dans la nef. Une niche est creusée à l'Est, sans doute à la tête du mort. Le plafond de la tombe est couvert de grands losanges à double bord (blanc et ocre rouge), enserrant des groupes de petits losanges pointés ; le tympan supérieur est orné de frises de bâtons rompus en demi-cercles. La douelle de l'arc est couverte de carrés imitant un décor de caissons en damiers. Tous ces ornements de remplissage se retrouvent dans les églises du cañon d'Ihlara (Yeşilköy) ${ }^{10}$.

Au pied de la tombe, s'élève une grande croix latine jaune pourvue des gemmes rouges et noires et entourée d'une auréole polylobée de styles " gréco-oriental ", enrichie d'ocelles rouges dans les angles (Fig. 5). En face, une croix sous arcade, dont les bras sont cernés de blanc et de rose, est située sous un grand quatre-feuilles, vert dans un carré jaune. Là encore, on retrouve le vocabulaire des églises du Haut Moyen Âge, dont Yılanlı kilise ${ }^{11}$.

La scène des cavaliers a été peinte sur une grande croix sous

\footnotetext{
${ }^{9}$ Nous remercions ici de son accueil M. Ramazan Yıldırım, Gouverneur du district de Güzelyurt ; intéressé aux antiquités de la région, il compte assurer l'entretien et la fermeture de l'église.

${ }^{10}$ Thierry, Nouvelles églises, Y1lanlı kilise, Kokar kilise, pl. 46, 63. Thierry, La Cappadoce, pl. 55-59.

${ }^{11}$ Thierry, Nouvelles églises, pl. 54. M. Restle, Die byzantinische Wandmalerei in Kleinasien, Recklinghausen 1967, fig. 475, 498. C. Jolivet-Lévy, Les églises byzantines de Cappadoce. Le programme iconographique de l'abside et de ses abords, Paris 1991, pl. 169, 170. Thierry, La Cappadoce, pl. 47-59.
} 


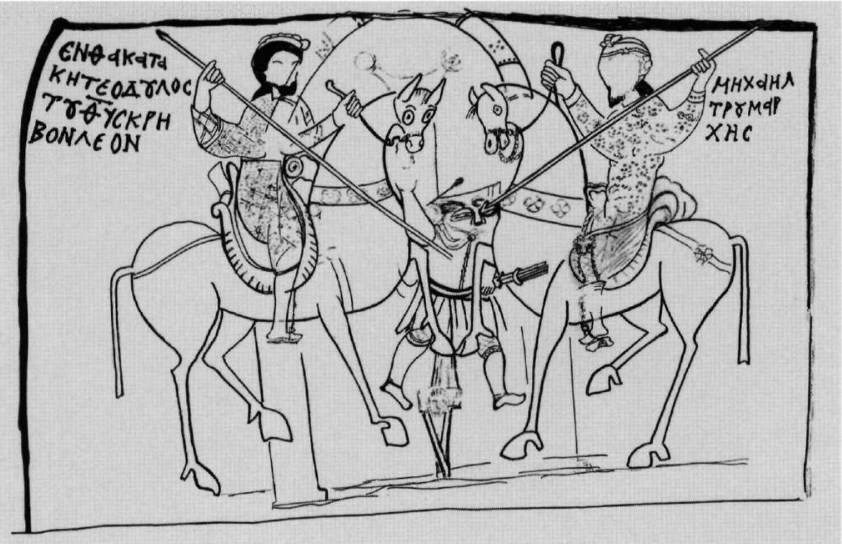

Fig. 2. Le combat du scribone et du tourmarque contre le démon (N.Thierry 2008).

arcade qu'on distingue mal (Fig. 2 et 5). La zone centrale, avec le croisement du bras supérieur et du bras latéral droit de la croix et, plus bas, avec les pattes levées des chevaux et les lances jaune pâle des attaquants, est difficile à analyser. Détériorations et repeints sont nombreux, si bien que les illustrations en noir et blanc sont peu lisibles. En zones saines, le logiciel de traitement des couleurs nous a permis de préciser certains détails, tout en renonçant parfois à identifier plusieurs tracés superposés. Notre schéma ne peut donc prétendre qu'à la compréhension du sujet.

\section{La scène du combat}

Les inscriptions qui nomment les cavaliers, deux adultes jeunes, sont peintes en ocre rouge, comme le cadre grossièrement tracé. Elles sont bien conservées et l'alphabet est conforme à celui des églises d'Ihlara de la fin du IXe siècle et du début du $\mathrm{Xe}^{12}$.

1. À gauche, sur le cheval noir, le scribone Léon est accosté de son épitaphe (Fig. 2 et 4): EN@A KATAKHTE $O \triangle O Y$ $\triangle O C$ TOY $\Theta(E O) Y$ CKPHBON $\triangle E O N$ (Ici repose le serviteur de Dieu, le skribone Léon).

Nous n'avions pas encore rencontré le titre de scribone (du latin scriba). Les scribones, officiers des excubiteurs (gardes

\footnotetext{
${ }^{12}$ Thierry, Nouvelles églises, fig. 51, col. $11,12,13$, et 15 (cette dernière, Eğri Taș kilisesi datant de 921-944).

${ }^{13}$ L. Bréhier, Les institutions de l'empire byzantin, Paris 1949, 187 (note 4, J.-B. Bury, The Imperial Administrative System, Londres 1911, 57-5861). A. H. M. Jones, Le déclin du monde antique, 284-610, coll. Histoire de l'Europe, I, 1970, 208. Nous remercions ici Georges Kiourtzian de ses indications.
}

du palais dont les effectifs augmentèrent au IXe siècle), avaient un rôle important comme envoyés de missions de confiance du basileus ${ }^{13}$. Ici, Léon, sans doute chargé de vérifier l'organisation stratégique d'une zone de guerre, avait été entraîné lui-même dans un combat mortel. De la main gauche, appuyée sur l'encolure, il semble vouloir retenir son cheval, alors que le démon le tirait à lui, la main droite levée. Le scribone perce de sa lance le thorax du démon. Ce coup est mortel, mais on peut penser qu'ici, il y a aussi une valeur magique décrite dans le Testament de Salomon, qui conte qu'en frappant la poitrine du démon avec l'anneau de Salomon, on lui faisait perdre sa force au point de le traîner comme un esclave ${ }^{14}$.

Léon portait une tunique longue à manches ajustées et un pantalon pris dans des bottes fines, costume de tradition orientale, avec des tissus à ornements géométriques et des semis ${ }^{15}$. Ses cheveux sont retenus par un double bandeau enroulé autour de la tête et noué sur le côté. Coiffure et costume ne sont pas d'un soldat byzantin en exercice, comme on le voit pour Jean Tzimiskès et Mélias dans le Grand pigeonnier de Çavuşin, tous deux représentés au retour de la campagne victorieuse de 965 (Fig. 9) ${ }^{16}$.

L'équipement du cavalier est particulier. La selle est suréle-

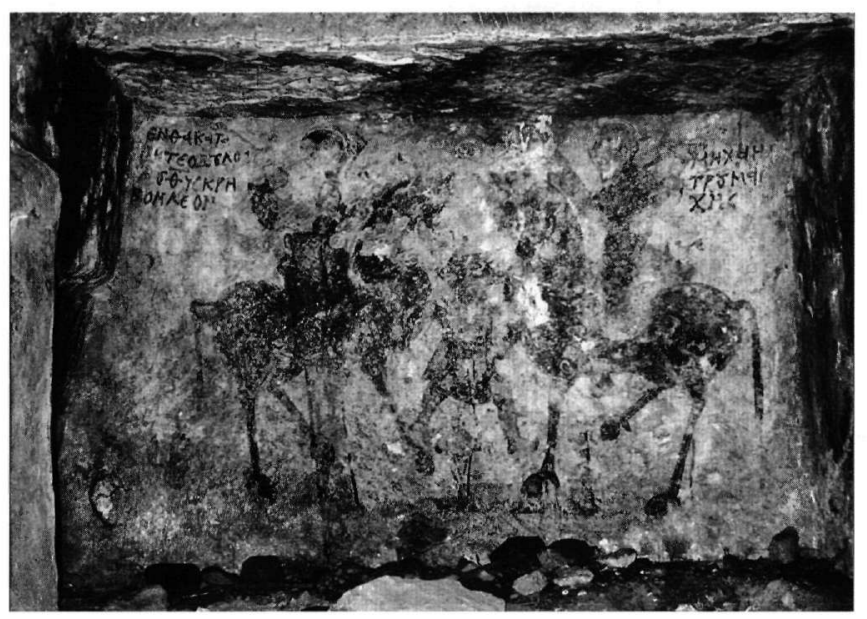

Fig. 3. Gelvere. L'arcade funéraire, vue générale.

14 P. Perdrizet, Negotium perambulans in tenebris, Strasbourg-Paris 1922, 32-33.

${ }^{15}$ Là encore nous reconnaissons le décor des vêtements de Georges et Théodore à Y1lanlı kilise (Fig. 4).

${ }^{16}$ N. Thierry, Haut Moyen Âge en Cappadoce, I, Paris 1983, 48-51. Ead., La Cappadoce, fiche 36. 


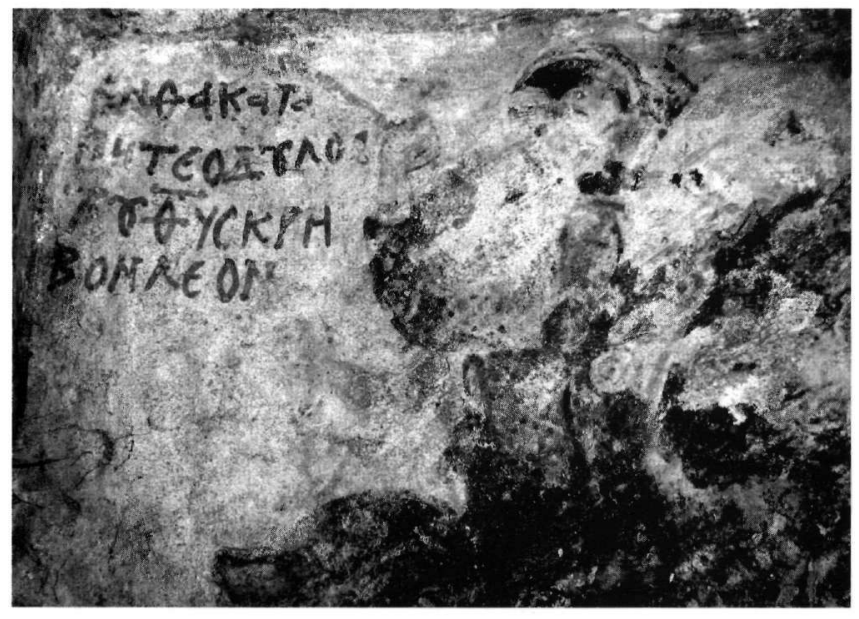

Fig. 4. Le scribone Léon.

vée et étroite, engainant le corps jusqu'à la taille, par des renforcements en avant et un dosseret en arrière. Excepté à Yllanlı kilise d'Ihlara, nous n'avons pas rencontré ce type de selle appropriée à la guerre équestre en montagne. On la retrouve chez les peuples nomades turco-mongols, et pour les troupes d'archers montés ${ }^{17}$.

L'étrier est court et du type à plancher. Reproduit avec précision sur le portrait du tourmarque, il rappelle l'importance de cet accessoire qui « marqua un tournant dans les pratiques du combat à cheval, surtout dans le maniement de la lance $»^{18}$. Il fallait que le combattant fit corps avec son cheval dans des actions dont le succès dépendait de la rapidité. Cette période des guerres des akrites ne nous a laissé aucune image comparable ${ }^{19}$.

2. À droite, se trouve le tourmarque Michel, sur un cheval rouge (Fig. 2 et 5). Il est simplement nommé : $M H X A H A$ TPOYMAPXHC (Michel troumarque, pour tourmarque) ${ }^{20}$.

Il tient haut les rênes souples et perce l'œil gauche du diable. La pointe de la lance pénètre dans l'angle interne.

${ }^{17}$ J.-P. Digard, « La culture équestre à l'origine », Chevaux et cavaliers arabes dans les arts d'Orient et d'Occident, Catalogue de l'Exposition de l'Institut du monde arabe, Paris 2003, 25-27.

${ }^{18}$ Exemple ottoman, op.cit., $110-112 \mathrm{n}^{\circ} 46$. Sur la guerre de montagne, Dagron, op.cit. (n. 6), 72-74, et 236.

${ }^{19}$ Les scènes équestres du manuscrit du Skylitzès de Madrid, dont les miniatures byzantines copient un manuscrit du XIe siècle, n'apportent rien de comparable; elles présentent « des groupes de cavaliers en armure, hérissés de lance », cf. A. Grabar, M. Manoussacas. L'illustration du manuscrit de Skylitzès de la B. N. de Madrid, Venise 1979, 173-177, 79-80.

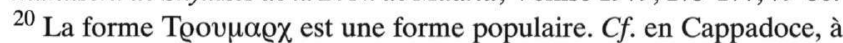
Ihlara, à Egri Taş kilisesi (921-944), Thierry, Nouvelles églises, 42. Thier-

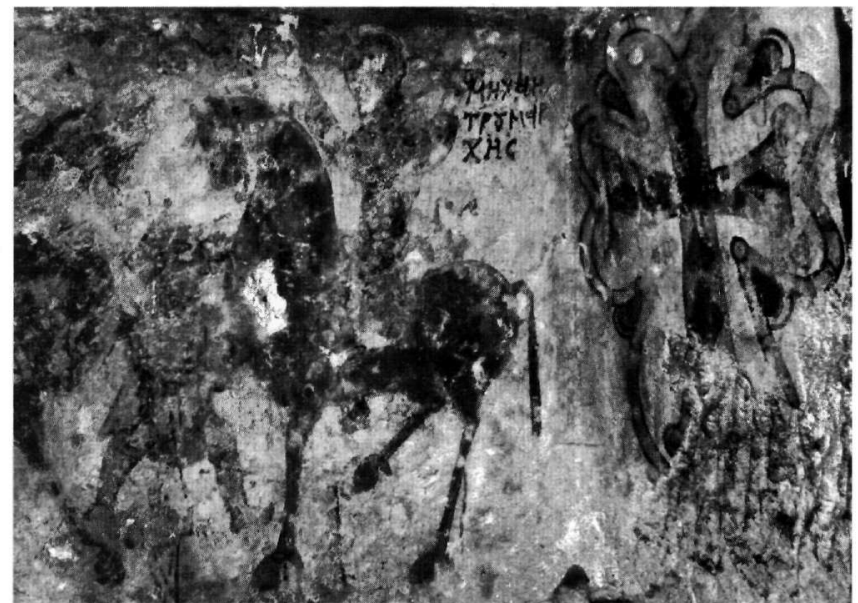

Fig. 5. Angle ouest de la niche funéraire.

On visait l'œil pour atteindre le cerveau et tuer d'emblé. Ici, cette attaque de l'œil du démon avait aussi valeur d'exorcisme $^{21}$. Il est vêtu d'une tunique décorée d'un semis de rosettes formées d'une perle dans une couronne d'autres plus petites. Le pantalon, paraît ample et souple, pantalon bouffant, évoquant celui des Parthes et des Scythes ; ce vêtement est resté en usage dans les provinces actuelles de l'Anatolie orientale et de la Syrie-Mésopotamie ${ }^{22}$. Le pied est glissé dans l'étrier à plancher.

L'altération des peintures ne nous permet pas d'identifier l'ornementation antérieure de la selle. Le pommeau est arqué comme parfois chez les Sassanides ${ }^{23}$. Plus bas, un disque orné d'une tête de lion paraît attaché à une fine ceinture passée en dessous de la taille du cavalier, et fixé à un harnais orné de perles qui descend devant sa jambe, peut-être pour la retenir sur la selle et relier l'étrivière $(\text { ? })^{24}$. Derrière la selle, un dosseret était sans doute destiné à retenir le paquetage lors des interventions courtes ${ }^{25}$.

Enfin, la coiffure du tourmarque est remarquable. C'est un

ry, La Cappadoce, fiche 31

${ }^{21}$ Perdrizet, op.cit. (n. 14), 28-31, sur l'exorcisme contre l'oculus invidiosus.

${ }^{22}$ Cf. H. Seyrig, "Armes et costumes iraniens de Palmyre », Antiquités syriennes. Deuxième série, Paris 1938, 47-66.

23 B. Marshak dans Splendeur des Sassanides (224-642), Catalogue, Bruxelles 1993, $\mathrm{n}^{\text {os }} 49,50,54$.

${ }^{24}$ Splendeur, op.cit., B. Overleat, 93, no 45 ; et Marshak, fig. 59, 94, 102. Nous n'avons pas dessiné les ornements possibles de la croupière (sch.1).

${ }^{25}$ Dagron, op.cit. (n. 6), Texte du traité sur la guérilla, 56-74, 92, 120. Commentaires, 145-49, 190, 215-231. 
léger turban de tissu blanc rayé de noir, dont l'extrémité est fixée sur le haut de la tête par un lien rouge. Cette partie haute du turban était ceinte d'un galon centré par une petite boucle étoilée. Le turban de faible volume caractérise les nobles arméniens du Vaspourakan au début du Xe siècle ${ }^{26}$. Plus important, il sera courant en Cappadoce au XIe siècle ${ }^{27}$. La mode venait du monde arabe où il existait déjà à l'époque préislamique, comme simple bandeau d'étoffe enroulé autour de la tête ${ }^{28}$. C'est ce que portait Constantin, lors du triomphe de son père Basile Ier, au retour de sa campagne victorieuse de Germanicée et Tephriké (878) : une « sorte de turban », blanc et tissé de fils d'or, « ayant sur le front comme une couronne d'or en lamé $»^{29}$.

Cette description fait penser à la coiffure du tourmarque Michel. Celui-ci est représentatif de son rang dans la hiérarchie régionale, second après le stratège et chef de la cavalerie ${ }^{30}$. À ce titre, bien que de solde élevée, il avait encore l'occasion de s'enrichir ${ }^{31}$.

3. Au centre, se tient le démon sous la forme d'un guerrier à tête de lion. Nous avons identifié d'emblée son nez aplati et court avec une arête nasale puissante encadrée de narines étalées. Son œil gauche est couvert en partie par la pointe triangulaire de la lance du tourmarque (Fig. 2 et 6 ).

En revanche, l'œil droit est difficile à découvrir à travers deux couches de repeints ${ }^{32}$. D'autant plus que sur le front, on a utilisé ce qui réapparaissait du bras latéral de la croix primitive pour peindre deux autres yeux en ligne supérieure ; à droite, il a été gratté (peinture et enduit). Des pratiques d'exorcisme expliquent, je pense, ces divers repeints et altérations de l'œil. Ce qui reste du masque du démon est du type léonin, tel que l'iconographie orientale l'a gardé des faces antiques de l'animal jusqu'aux schématisations abbassides puis seldjoukides et ultérieures ${ }^{33}$. Ce lion à tête ronde

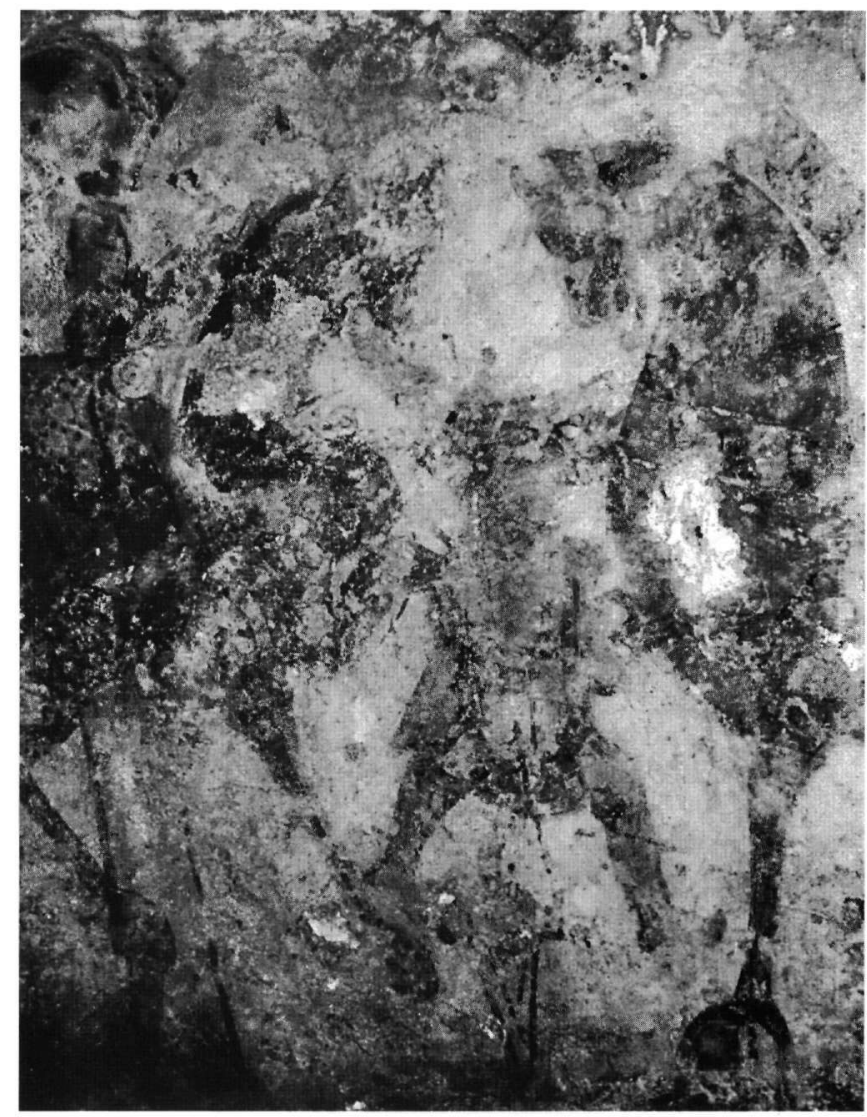

Fig. 6. Le démon entre les deux chevaux de ses adversaires.

a été adopté par les sculpteurs d'Arménie et de Géorgie, régions sensibles à la civilisation abbasside ; les bas-reliefs du porche de l'église géorgienne de Hahuli (en Turquie) sont exemplaires (Fig. 10) ${ }^{34}$.

Le démon porte une tunique courte et sa cuirasse est couverte

\footnotetext{
${ }^{30}$ Dagron, op.cit. (n. 6), division des thèmes, 73-74 ; privilèges, 269-272, rôle dans la guerre, 206-207, 216, 218, etc (index, 351).

31 Op.cit., Commentaires, 233-234. Dans sa harangue aux troupes, Constantin VII énumère les récompenses impériales des hauts faits, $\mathbf{H}$. Ahrweiler, «Un discours inédit de Constantin VII Porphyrogénète », TM II (1967) (Reprints, London 1971, XII, 393-404, 400-401).

${ }^{32}$ Les essais de surexposition de la photo, effacent les surcharges et permettent de voir le faciès primitif, les deux yeux encadrant le nez.

${ }^{33}$ Ch. K. Wilkinson, « Assyrian and Persian Art », BMMA, March 1955, 213-224 avec figures. J. Gierlichs, Mittelalterliche Tierreliefs in Anatolien und Nordmesopotamien, Tübingen 1996, 84-91, pl. 33, 44, 48, 54 etc.

${ }^{34}$ D. Winfield, « Some Early Medieval Figure Sculptures from NorthEast Turkey », JWarb XXXI (1968), 58-65, fig. p. 61, 63 (du règne de David Curopalate, 972-1001).
} 


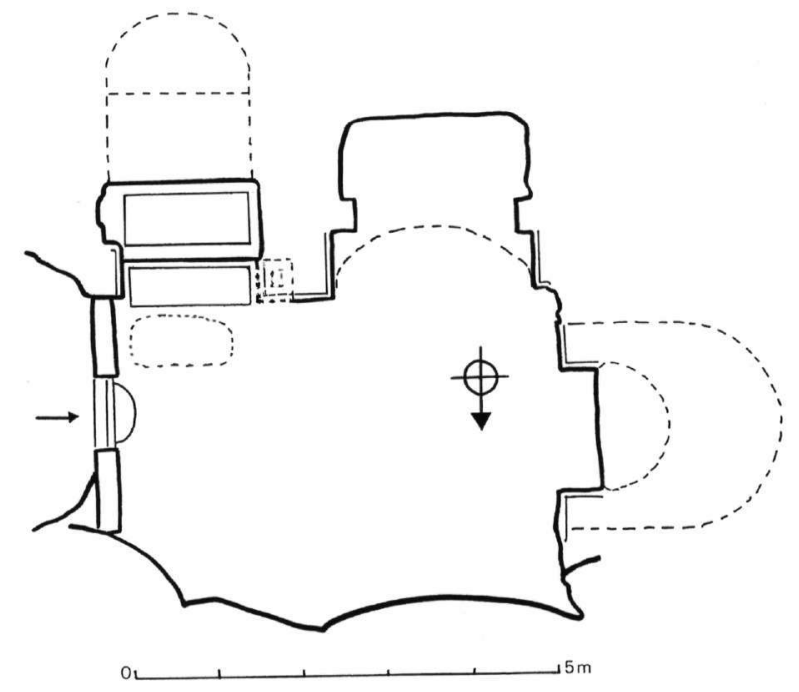

Fig. 7. Gelvere. Plan de l'église rupestre (N.Thierry 2008).

d'un surcot fermé par des nœuds sur la ligne médiane ${ }^{35}$. Ses jambes sont moulées par des bottes serrées sous les genoux. Le manteau gris-bleu est rejeté en arrière par son bras droit levé vers la tête du cheval du scribone pour saisir le mors.

Il est tourné vers le tourmarque. Il prend appui sur ses jambes et attaque le cheval avec une étrange arme de poing, ressemblant à une petite fourche à trois longues lames fines et serrées montées sur la garde d'un manche de poignard. Elle s'apparenterait au katar, arme meurtrière car les lames sont larges et à pointes renforcées, deux latérales et une centrale jaillissant lorsqu'on serre le manche. Nous n'avons pas trouvé d'équivalent, sauf dans la littérature épique orientale $^{36}$. Ce maniement de l'arme évoque les soldats partant à l'attaque, leur arme spécifique à la main ${ }^{37}$.

Le lion est connu comme symbole du démon. Déjà dans l'ancien Testament on lit «Sauve-moi de la gueule du lion »

\footnotetext{
${ }^{35}$ Accessoire « qui ne doît pas être blanc », pour passer inaperçu, Dagron, op.cit. (n. 6), 52-53, §4.

${ }^{36}$ Fr. Wilkinson, «Inde et Asie du sud-est », dans Épées et armes blanches, Paris 1990, 186-203, fig. p. 194-196 (p. 186 : « en combat rapproché, on utilisait des masses et des haches d'arme,... et il est fait mention d'une dague de poing, la maustika, qui évoque vaguement l'arme plus récente dite katar); A. C. Tirri, Islamic Weapons. Maghrib to Moghul, Librairie du Congrès, Washington 2003, 73 et fig. 45 (variétés de copies d'armes de Perse, où figurent des armes composites, à trois couteaux jumelés, et un petit trident sur manche à trois couteaux). Autre exemple iranien de trident à lames minces et serrées, comme extrémité de javelot ou de lance, dans G. Cameron Stone, A Glossary of the Construction, Decoration and Use of Arms and Armor, New York 1961, 629 , fig. $800, \mathrm{n}^{\circ} 1$; rappelons que Zulfikar, le sabre légendaire de Mahomet, avait deux pointes, p. 685. Nous remercions M. J.-P. Sage-Fresnay,
}

(Ps. 22, 22, repris par Paul, 2 Tim 4, 17), et Pierre ( I - 5, 8) : « Votre partie adverse, le Diable, comme un lion rugissant, rôde, cherchant qui dévorer ${ }^{38}$.

En bas de la scène, la grande croix est restée telle qu'on l'avait peinte antérieurement, menaçant le corps du démon ${ }^{39}$. Cette pensée obsédante du diable était très ressentie à Ihlara où, dans trois églises, il est présent à la Cène, bravant le Christ ${ }^{40}$.

\section{Commentaires}

La composition caractérise la région des guerres frontalières et leur époque. Elle rend hommage à ces combattants, «morts à la guerre pour l'empereur et les Chrétiens ».

La scène rappelle un Office qui leur était dédié et que les éditeurs datent des premières décennies du Xe siècle ${ }^{41}$. Les formules se répètent : « ... célébrons la mémoire / de nos frères qui moururent en combattants... vénérons-les, que cela serve / au rachat de leur âme... (f. 12r, 7-13).

Les Tactica de Léon VI détaillaient les cérémonies religieuses qui assuraient la pureté des soldats qui partaient au

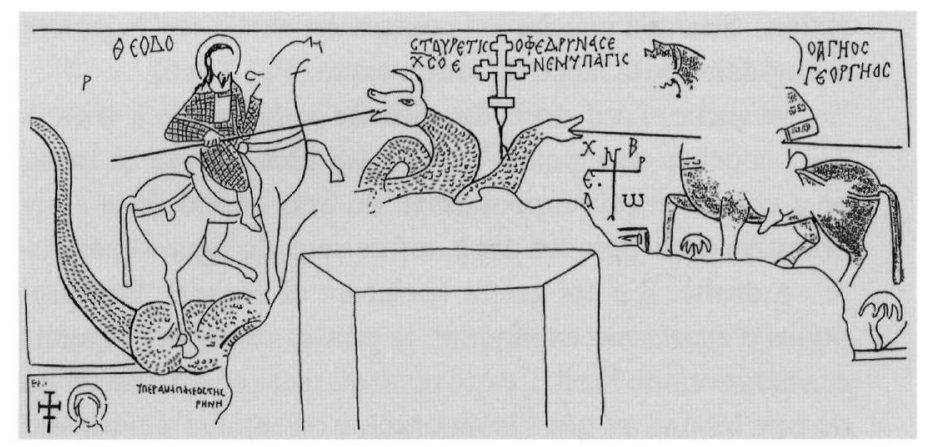

Fig. 8. La croix victorieuse de l'entrée d'Yilanlı kilise, à Ihlara (N. Thierry 1999).

conservateur au Musée de l'Armée, et sa collaboratrice, qui m'ont aidé pour cette enquête ardue.

${ }^{37}$ Dagron, op.cit. (n. 6), 52-53, §4.

38 X.R. Mariño Ferro, Symboles animaux (éd. de Brouwer), 1996, 204, notes p. 457.

${ }^{39}$ On pense au serpent percé par la croix (sch. 2), comme Hadès perforé par le pied de la croix sur le célèbre ivoire du Metropolitan Museum (dans Thierry, op.cit. (n. 2), 235 et fig. 3).

${ }^{40}$ Thierry, La Cappadoce, 158-159. A Yllanlı kilise, en particulier, le sujet est développé, et le diable nommé : un démon Céléphouzé est derrière le Christ et le démon « de la désobéissance " provoque Jésus.

${ }^{41}$ Th. Détorakis et J. Mossay, « Un office byzantin inédit pour ceux qui sont morts à la guerre, dans le Cod. Sin. Gr. 734-735 », Le Muséon 101 (1988), 183-211. 


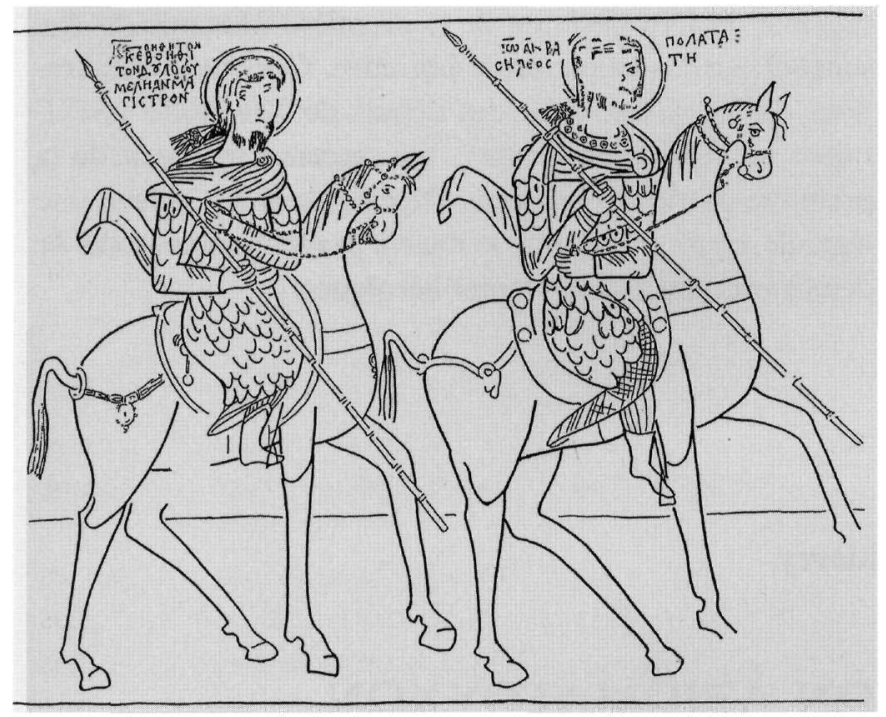

Fig. 9. Jean Tzimiskès et Mélias à Çavuşin, ca $965-969$ (N. Thierry 1983).

combat, justifiant ainsi leur accès au paradis. Le « tabou du sang versé » était négligé ${ }^{42}$.

Les empereurs, insistaient sur la notion de Guerre Sainte pour galvaniser les troupes, s'opposant ainsi au djihâd des Arabes $^{43}$.

Nicéphore Phocas (comme Héraclius) aurait décidé « que les soldats morts au combat soient honorés à l'égal des martyrs et célébrés par des hymnes semblables aux leurs », si le clergé ne s'y était opposé. Mais un monument cappadocien, l'église de Nicéphore à Çavuşin illustre la reconquête comme une mission divine et, peu après la mort de l'empereur, l'Église de Constantinople lui consacra un office liturgique ${ }^{44}$.

On a remarqué que les deux cavaliers de Gelvere ne sont pas nimbés, ce signe de distinction étant employé ailleurs pour les membres de la famille impérial et héros célèbres (Fig. 4) ${ }^{45}$.

On a noté tous les traits orientaux et régionaux de cette peinture, témoin des thèmes frontaliers ${ }^{46}$. Gelvere faisait partie d'un de ces petits thèmes qui contrôlaient les défilés menant aux Portes de Cilicie longtemps tenues par les

\footnotetext{
${ }^{42}$ Béatrice Caseau, J. C. Cheynet, « La communion du soldat et les rites religieux sur le champ de bataille ", Mélanges offerts à Pierre Maraval (Histoire et Civilisation de Byzance, 23), Paris 2006, 114-119.

${ }^{43}$ Dagron, op.cit. (n. 6), « Un manifeste en faveur des soldats » et « Nicéphore Phocas et l'Armée », 259-287.

44 Thierry, La Cappadoce, 73, 173-177. B. Flusin et J.-Cl. Cheynet, Jean Skylitzès, Empereurs de Constantinople, Paris 2003, 230-31.

${ }^{45}$ Cf. n. 16 et Marthe Collinet-Guérin, Histoire du nimbe, Paris 1961, 315-436.

${ }^{46}$ Dagron, op.cit. (n. 6), « La frontière et les frontaliers », 239-257.
}

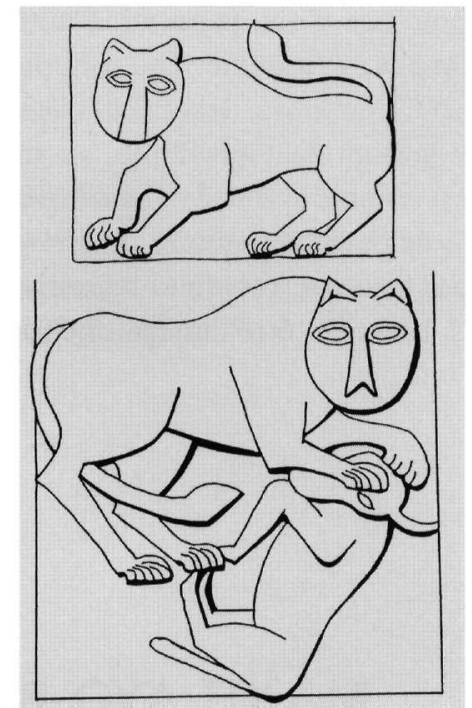

Fig. 10. Types de lions du porche sud de Hahuli, 2e moitié du Xe s. (D. Winfield 1968).

Arabes, « petits thèmes, désignés du nom de la ville ou de la forteresse qui leur servait de point d'appui et étaient commandés en partie par des nobles arméniens $\gg{ }^{47}$.

Nous avons cité souvent le groupe des cinq églises groupées près du village d'Ihlara, caractérisées par des éléments étrangers au monde constantinopolitain. L'une, Eğri Taş kilisesi, datée de Romain Lécapène (921-944), était la fondation d'un spatharocandidat et tourmarque de Spadiata et Pates, le premier site localisé à environ $120 \mathrm{~km}$ au sud ; l'imagerie reflétait des sources irano-syrienne ${ }^{48}$. La seconde, Ağaç altı kilise (fin VIIIe-IXe s.), a conservé des peintures d'un art post-sassanide et arabisant ${ }^{49}$. Trois autres, apparentées et datables du IXe au Xe, traduisent un art de schématisation rude et une inspiration religieuse atypique, reflétant l'usage des apocryphes, une pensée dualiste et monophysite, il s'agit d'Yılanlı kilise, Kokar kilise et, plus atténuée et orthodoxe, Pürenli seki kilisesi ${ }^{50}$. De celles-ci, la première est

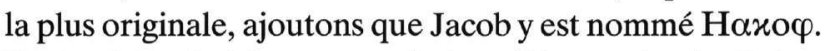
En bref, les établissements de la vallée proche de Gelvere

\footnotetext{
${ }^{47}$ Dagron, op.cit., 244. J.-C. Cheynet, G. Dédéyan, « Militarisation de l'Empire et intégrations des Arméniens » dans Histoire du peuple arménien, Toulouse 2007, 300-313, 315-317.

${ }^{48}$ Thierry, Nouvelles églises, 39-72, compléments. Thierry, La Cappado$c e$, fiche 31, pl. 59-62. Irène Beldiceanu-Steinherr, « Une tourma révélée par l'inscription de l'église E. T. », JÖB 38 (1988), 395-420.

49 Thierry, Nouvelles églises, 73-87. Thierry, La Cappadoce, 140-142, fiche 23, pl. 52-55.

50 Thierry, Nouvelles églises, 89-153. Thierry, La Cappadoce, 154-163, fiches 32, 33, pl. 55-59.
} 
traduisent la présence d'une population hétérogène d'installation ancienne. Les Arméniens ne sont pas directement présents, mais, à Kokar kilise, ils sont cités comme habitants de la province voisine, la Gabadonie, au sud de Kayseri (Barthélemy, est nommé apôtre ẻv $\Gamma \alpha \beta \alpha \delta o v i \alpha)^{51}$.

Il reste difficile de dater la peinture de Gelvere qui se rattache évidemment aux guerres arabo-byzantines et aux combats des akrites. Elle peut être l'image célébrant un combat régional du IXe siècle ou de la première décennie du Xe, substituée à la grande croix primitive. Quelques restaurations du Xe siècle, voire des débuts du XIe siècle traduiraient un vénération locale $\mathrm{e}^{52}$. On retrouve le climat de la première partie du roman de Digénis Acritas. Comme une légende, ce combat contre le Diable perpétue le souvenir de deux morts glorieux des temps héroïques.

\author{
Nicole Thierry
}

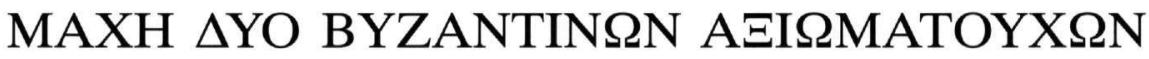 $\Sigma$ TA $\Sigma$ YNOPA TH $\Sigma$ KAППА $\triangle$ OKIA $\Sigma$

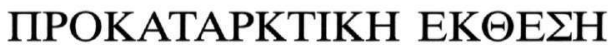

$\Sigma$

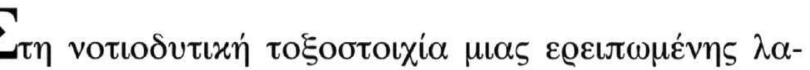

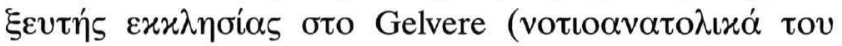

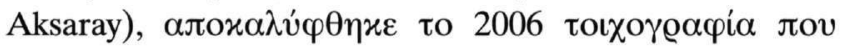

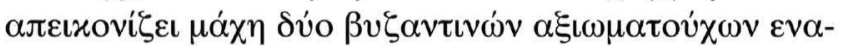

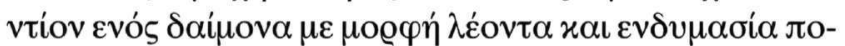

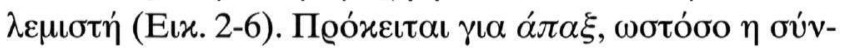

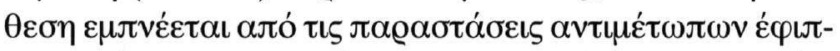

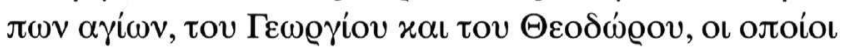

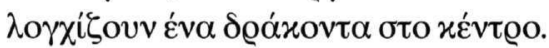

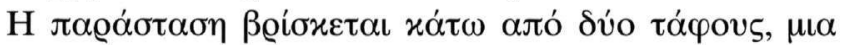

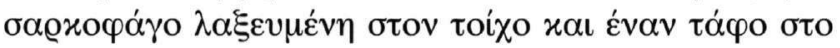
$\varepsilon \dot{\varepsilon} \alpha \circ \varsigma, \mu \pi \varrho \circ \sigma \tau \alpha \dot{\alpha} \tau \zeta$.

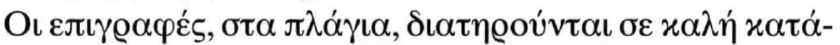

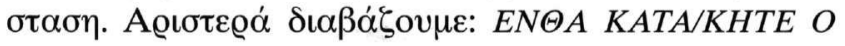
$\triangle O Y A O C / T O Y \Theta(E O) Y$ CKPH/BON $A E O N$ (Eıx. 2 xal

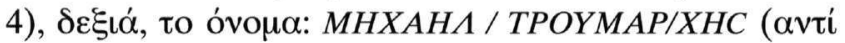

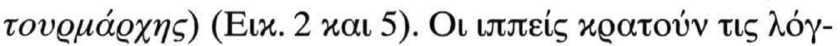

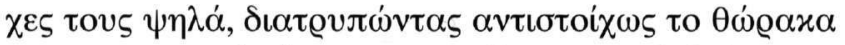

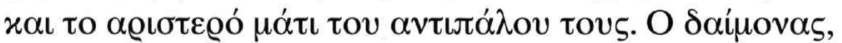

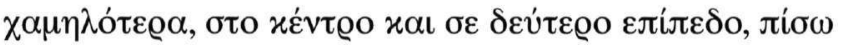

\footnotetext{
${ }^{51}$ Comprise dans la Petite Arménie, avec les terres entre Césarée et Mélitène, en raison d'un peuplement connu depuis le VIIe siècle, Thierry, Nouvelles églises, 129. A Göreme, dans l'église St-Jean de Güllü Dere (913-920), c'est Thaddée qu'on cite « en Gabatho Grande Arménie », Thierry, La Cappadoce, 160-161, pl. 75.
}

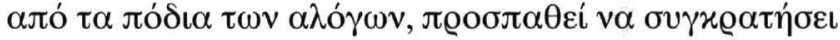

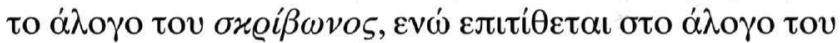

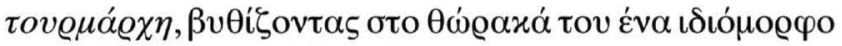

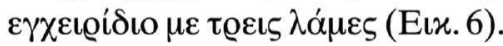

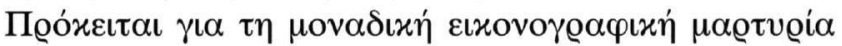

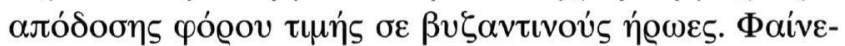

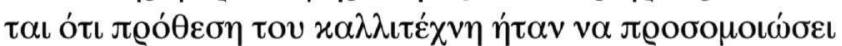

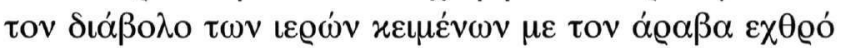

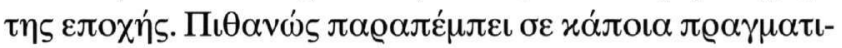

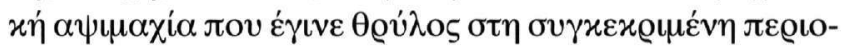

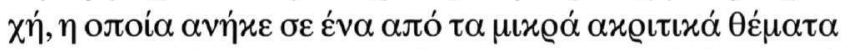

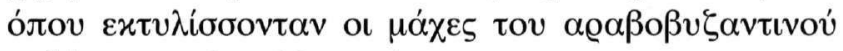

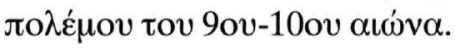

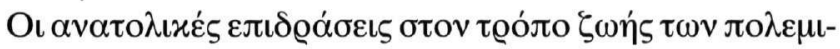

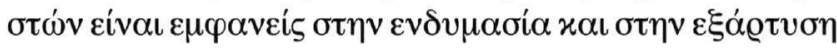

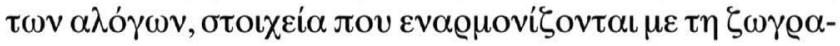

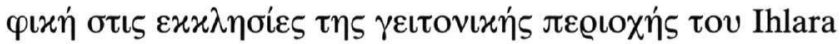

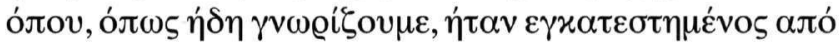

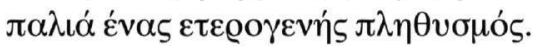

\footnotetext{
${ }^{52}$ Citons des épitaphes, modestes mais exposées au public, sur la façade et à l'entrée d'églises dans le Kara Dağı : celle d'un Philaretos « mort à la guerre » (ca 750-770) et d' un Mousianos « qui endura de nombreuses blessures » (après 900), W. M. Ramsay et Miss G. Bell, The Thousand and one Churches, Londres 1909, 545-546, 525-526, 537.
} 